\title{
EVOLUZIONE DELLA LINGUA E STATICITÀ DELLA NORMA NELL'TTALIANO CONTEMPORANEO: GLI AUSILIARI NEI COSTRUTTI CON VERBI MODALI
}

\section{UN ESEMPIO DEL MOVIMENTO DELL'ITALIANO CONTEMPORANEO?}

Nel 2007 due studi si sono occupati dell'ausiliare utilizzato nei costrutti con verbi modali (potere, dovere, volere). Telve (2007) ha cercato di ricostruire la distribuzione degli ausiliari in tali costrutti in un arco di tempo che va dalle Origini all'Ottocento (con qualche accenno alla contemporaneità); io stesso, in un saggio che richiamo anche nel titolo (Cortelazzo 2007a), ho citato il caso dell'ausiliare con i verbi modali come un palese esempio di scarto tra la vulgata grammaticale, quale emerge nelle grammatiche scolastiche o negli interventi divulgativi sulla lingua italiana, e la trattazione scientifica della grammatica dell'italiano, secondo le impostazioni più adeguate alla realtà empirica e ai principi teorici paradigmatici.

Detta in poche parole, la questione è questa: a proposito dell'ausiliare da utilizzare nei tempi composti dei verbi modali (seguiti da un infinito), la tradizione grammaticale, a partire da Bembo, sostiene che la norma corretta, anche se non sempre rispettata, consista nel ricorso all'ausiliare del verbo all'infinito e non a quello proprio del verbo modale, cioè avere (quindi «Michele sarebbe dovuto tornare a casa» e non «Michele avrebbe dovuto tornare a casa»). L'uso comune, che contempla spesso il ricorso ad avere anche con i verbi inaccusativi (cioè i verbi non transitivi che richiedono l'ausiliare essere), sarebbe quindi un uso contrario alla norma.

Questa asserzione ha tre aspetti che poche volte sono stati sottoposti a verifica. Il primo riguarda la convinzione che si tratti semplicemente di una questione di norma. Le due costruzioni sarebbero grammaticalmente equivalenti e l'utilizzo dell'ausiliare avere anche con verbi inaccusativi dipenderebbe semplicemente da un dominio poco sicuro, da parte di alcuni parlanti o scriventi, della norma dell'italiano: basti pensare che Coletti (1993: 320) ascrive ai «problemi con l'italiano» di Italo Svevo e alla sua «competenza approssimativa» l'uso dell'ausiliare avere «specie in presenza di verbo servile» (che regge un verbo inaccusativo, s'intende).

La seconda consiste nella convinzione, spesso data come implicita, che la sostituzione dell'ausiliare essere con avere sia un'innovazione dell'italiano contemporaneo. $\mathrm{Si}$ tratterebbe, quindi, di uno dei tratti del processo di ristandardizzazione dell'italiano che si sta realizzando negli ultimi decenni. ${ }^{1}$

\footnotetext{
*Indirizzo dell'autore: Facoltà di Lettere e Filosofia, Piazza Capitaniato 7, 35139 Padova, Italia. Email: cortmic@unipd.it

${ }^{1}$ Anche Tavoni (2002: 147) si occupa della collocazione dei modali all'interno dei processi di ristandardizzazione dell'italiano, ma lo fa da un altro punto di vista (sia pure strettamente collegato al fenomeno che stiamo discutendo in questo contributo): la risalita del clitico con verbi modali in costrutti come Non ci posso credere invece di Non posso crederci.
} 
La terza ha a che vedere con la mancanza di dati assodati sulla distribuzione dei due ausiliari nei costrutti presi in esame, sia nella storia dell'italiano, sia nella fase contemporanea.

Per il primo punto, gli studi più recenti (come si può ricavare da Renzi/Salvi/Cardinaletti 2001: 514-522), tenendo conto di più di un comportamento dei costrutti con verbi modali, giungono a una conclusione diversa. Quello che nella superficie della frase individuiamo come un unico costrutto con due possibili realizzazioni è invece la rappresentazione superficiale apparentemente identica di due costruzioni diverse: una formata dal verbo modale con una proposizione oggettiva («Io voglio mangiare una pastasciutta» con una struttura del tutto analoga a «Io voglio una pastasciutta») e una in cui il verbo modale forma con il verbo all'infinito un unico complesso verbale (in questo caso «Io voglio mangiare una pastasciutta» va interpretato con «voglio mangiare» come complesso verbale e «una pastasciutta» come oggetto diretto del verbo mangiare unito a voglio; è il processo che va sotto il nome di ristrutturazione). Per l'aspetto che ci riguarda la differenza tra i due costrutti si evidenzia quando il verbo che si accoppia al modale è un verbo che nei tempi composti ha l'ausiliare essere: se la forma realizzata è quella del verbo modale che regge una proposizione oggettiva, l'ausiliare sarà quello del verbo modale (quindi, avere: "Gianni ha voluto andare a tutti i costi al cinema», con «andare a tutti i costi al cinema» interpretabile come proposizione oggettiva); se invece la forma realizzata è quella con ristrutturazione, l'ausiliare sarà quello del verbo all'infinito (quindi, essere: "Gianni è voluto andare a tutti i costi al cinema» analogamente a »Gianni è andato al cinema «). Insomma, non si tratta di una semplice scelta tra una norma più formale e una più informale, ma di un'opzione tra due strutture grammaticali diverse; anche se non vi è dubbio che l'opzione tra una struttura e l'altra dipende, almeno in parte, dal grado di formalità del testo.

Sul secondo punto, la ricerca di Telve (2007) ha portato, con il conforto di dati fattuali, qualche chiarezza. Se nel Due e Trecento possiamo davvero dire che prevale l'ausiliare essere, soprattutto negli scrittori toscani, nei secoli successivi essere si alterna sempre più frequentemente con avere (soprattutto negli scrittori non toscani), fino a quando, nel Settecento, avere prende decisamente il largo (Goldoni, per es., non usa mai essere). La situazione che riscontriamo nel Novecento, dunque, non è che il punto di approdo di un movimento che ha radici ben lontane nell'evoluzione della nostra lingua; se a qualcuno, ancor oggi, l'uso dell'ausiliare avere pare un dato innovativo o un uso da stigmatizzare è perché la lezione bembiana (che anche in questo caso era direttamente legata all'uso trecentesco toscano) ha condizionato il nostro modo di vedere la norma molto più a lungo di quanto abbia potuto condizionare il nostro uso: ${ }^{2}$ al giorno d'oggi anche divulgatori brillanti e disinvolti come Beppe Severgnini (2007: 176) considerano senza mezzi termini «sbagliato» l'uso dell'ausiliare avere davanti a un

\footnotetext{
2 Non è questo l'unico fenomeno nel quale innovazioni apparentemente moderne si rivelano semplicemente come manifestazioni pienamente mature di processi che rimontano molto indietro nel tempo; si veda, ad es., il caso della perifrasi progressiva (Cortelazzo 2007b), che vede un uso incipiente già nel Quattrocento e un suo progressivo e decisivo allargarsi a partire dal Settecento.
} 
modale quando regge un verbo che nei tempi composti richiede l'ausiliare essere. Del resto, il fenomeno è uno di quelli segnati dalla penna correttrice di Satta (1989).

Sul terzo punto, sono molti i linguisti, o gli appassionati di lingua, che hanno azzardato opinioni sulla frequenza d'uso dei due ausiliari. Già nel 1964 Franco Fochi (1964: 278) osservava che «il linguaggio familiare va meno per il sottile, e adopera quasi sempre avere». Più recentemente Paolo D'Achille (2003: 116), parlando in generale dell'italiano, senza distinzioni di livello, ha sostenuto che essere «sembra largamente preferito» ad avere (anche se, aggiunge, nella frase non ho potuto essere presente l'uso di avere «è da considerare senz'altro corretto»), mentre Serianni (2006: 145) afferma, sia pure con accorta prudenza, che «a quanto sembra, avere è in espansione».

Sono tutte prospettive che si basano sulle sensazioni degli studiosi e non su riscontri oggettivi (e questo spiega la diversità quanto meno di accenti). A nulla sono valsi i risultati presentati da Boysen (1977), ampiamente utilizzati da Skytte (1983: 95-103): si tratta di una ricerca fondata su una solida base documentaria (un corpus di 10.000 pagine di narratori italiani del dopoguerra), eccezionalmente ampia per gli anni in cui è stata raccolta. ${ }^{3}$

\section{UN'INDAGINE SULL'ITALIANO CONTEMPORANEO}

Nonostante l'esistenza dello studio di Boysen (1977) credo, quindi, che sia opportuno dar seguito al suggerimento di Serianni (2006: 145), secondo il quale i dati «dovrebbero essere confermati da indagini specifiche (per esempio sulla lingua dei giornali o su campioni di italiano parlato)». È quanto sto facendo per cercare di avere un quadro documentato sugli usi attuali della nostra lingua, soprattutto per quel che riguarda i fenomeni di ristandardizzazione, o di presunta ristandardizzazione, dell'italiano. L'obiettivo è quello di avere un corpus di riferimento, sul quale condurre osservazioni quantitative e qualitative sui singoli fenomeni.

Per il momento, non avendo ancora a disposizione un corpus sufficientemente ampio e variegato di articoli giornalistici (che sto attualmente allestendo), utilizzo solamente i corpora messi a disposizione degli studiosi di italiano contemporaneo, con generosità e lungimiranza, da Tullio De Mauro: innanzitutto il corpus di narrativa dal dopoguerra ad oggi pubblicato con il titolo di il Primo Tesoro della Lingua Letteraria Italiana del Novecento. ${ }^{4}$

\footnotetext{
${ }^{3}$ Noto, per inciso, che, ancora una volta, uno studio che cerca di descrivere l'effettivo andamento di un fenomeno dell'italiano, basandosi sullo spoglio di un campione di testi, è dovuto a uno studioso straniero (cfr. Cortelazzo 2007a: 47).

4 Il Primo Tesoro della Lingua Letteraria Italiana del Novecento, edito da UTET, è costituito da 100 romanzi degli anni 1947-1981, che hanno partecipato al Premio Strega: per ogni anno c'è il vincitore, più uno o due degli altri romanzi giunti in finale. Per le mie ricerche sto integrando il corpus con altri romanzi della seconda metà del Novecento; in questo studio ho spogliato anche Giuseppe Berto, Il male oscuro (1964), Italo Calvino, Le città invisibili (1972), Beppe Fenoglio, Una questione privata (1965), Dario Fo, Morte accidentale di un anarchico (1970), Carlo Levi, Cristo si è fermato a Eboli (1945), Primo Levi, Se questo è un uomo (1958) e La tregua (1963); Giorgio Manganelli, Hilarotragoedia (1964), Anna Maria Ortese, Il mare non bagna Napoli (1953), Pier Paolo Pasolini,
} 
Per un riscontro sul parlato sono ricorso al corpus del Lessico di frequenza dell'italiano parlato (De Mauro et alii 1993), ora interrogabile grazie al software della BAnca Dati dell'Italiano Parlato (BADIP) dell'Università di Graz (http://badip.uni-graz.at). Questo corpus, tuttavia, ha una dimensione non paragonabile a quello scritto appena citato e può servire solo da primo parziale confronto.

In entrambi i corpora ho raccolto tutte le occorrenze dei participi passati dei verbi potere, dovere, volere; tra i contesti così isolati, ho estratto quelli nei quali i tre verbi fungono da modali e reggono infiniti di verbi che richiedono l'ausiliare essere (o costruzioni verbali, come il passivo, che richiedono tale ausiliare).

Le variabili da prendere in esame sono state ben delineate da Telve (2007), Boysen (1977), Skytte (1983): innanzi tutto il modale utilizzato (i tre verbi modali non mostrano necessariamente tutti lo stesso comportamento), il verbo impiegato, l'origine geografica degli scriventi.

\section{LA SITUAZIONE NELL'ITALIANO LETTERARIO CONTEMPORANEO}

Nel corpus di letteratura italiana contemporanea, i casi utili per la nostra indagine sono 1937. Esaminando i risultati in maniera aggregata (tab. 1), risulta confermata la tendenza delineata in prospettiva storica da Telve (2007): il processo che ha portato a preferire l'ausiliare avere (e quindi a considerare l'infinito come componente di una proposizione infinitiva) è molto avanzato, dato che quasi tre quarti delle occorrenze esaminate (precisamente il 74,24\%) presentano l'ausiliare avere.

\begin{tabular}{|l|l|l|}
\hline ausiliare & valori assoluti & percentuale \\
\hline avere & 1438 & $74,24 \%$ \\
\hline essere & 499 & $25,76 \%$ \\
\hline totale & 1937 & $100,00 \%$ \\
\hline
\end{tabular}

Tab. 1 - Distribuzione, in valori assoluti e in percentuale, degli ausiliari

La situazione si presenta però diversificata in relazione al verbo modale implicato. Il quadro generale è rappresentato dalle tab. 2 e 3 :

\begin{tabular}{|l|l|l|l|}
\hline ausiliare & Potere & dovere & volere \\
\hline avere & $67,83 \%$ & $74,00 \%$ & $94,88 \%$ \\
\hline essere & $32,17 \%$ & $26,00 \%$ & $5,12 \%$ \\
\hline totale & $100,00 \%$ & $100,00 \%$ & $100,00 \%$ \\
\hline
\end{tabular}

Tab. 2 - Distribuzione in valori assoluti degli ausiliari in dipendenza dai tre verbi modali

Ragazzi di vita (1955) e Una vita violenta (1959), Cesare Pavese, La luna e i falò (1950), Lalla Romano, Tetto murato (1957), Elio Vittorini, Uomini e no (1945), Paolo Volponi, Memoriale (1962) e Il pianeta irritabile (1978). 


\begin{tabular}{|l|l|l|l|}
\hline ausiliare & potere & dovere & volere \\
\hline avere & 622 & 538 & 278 \\
\hline essere & 295 & 189 & 15 \\
\hline totale & 917 & 727 & 293 \\
\hline
\end{tabular}

Tab. 3 - Distribuzione in percentuale degli ausiliari in dipendenza dai tre verbi modali

Come si vede, il modale maggiormente implicato nei costrutti che ci interessano è potere, seguito da dovere e, a lunga distanza, da volere. Però, nella scelta dell'ausiliare la palma per il ricorso ad avere, scelta contraria alla norma, va ai costrutti imperniati su volere, seguito da dovere e poi da potere: insomma la maggior frequenza del verbo modale sembra garantire una maggiore presenza dell'ausiliare richiesto dalla norma (essere).

I risultati confermano sia le considerazioni di Telve (2007), che ha preso in considerazione soprattutto potere e volere, dato che per dovere l'impiego come modale è stato marginale fino al Seicento, sia quelle ricavabili dallo studio di Boysen (1977). Rispetto a quest'ultimo, va notato, tuttavia, che il nostro corpus mostra una decisa accelerazione del processo di utilizzo dell'ausiliare avere sia in generale, sia con i singoli modali. Il valore percentuale complessivo di uso di avere ricavabile dagli spogli di Boysen, infatti, è del 69,24\%, inferiore di 5 punti al valore ricavato dall'esame del corpus da me utilizzato. Inoltre, mostra, per così dire, il sorpasso di dovere su potere. Sommando tutte le tipologie di uso dei modali nei tempi composti in connessione con verbi inaccusativi, dallo studio di Boysen risulta che avere viene utilizzato nel $66,21 \%$ dei casi se il modale è potere, nel $65,61 \%$ se si tratta di dovere, nell' $82,50 \%$ se si tratta di volere. Se ne deduce che l'incremento complessivo notato è dovuto non tanto all'incremento dell'uso di avere con potere (che risulta aumentato di poco più di un punto e mezzo percentuale), quanto dall'uso con dovere (aumento di più di 8 punti percentuali) e con volere (aumento di 12 punti percentuali). Poiché la conformazione del corpus di Boysen è molto simile a quella del corpus utilizzato nel presente articolo, si deve ipotizzare che l'estensione dell'uso di avere abbia proceduto con grande velocità nell'ultimo quarantennio. ${ }^{5}$

Per quel che riguarda il peso che, nella scelta dell'ausiliare, può avere il verbo all'infinito (aspetto trattato sia da Boysen 1977 sia da Telve 2007), non mi sento in questa sede di proporre dati, vista la grande dispersione di verbi implicati nel costrutto e la loro differente distribuzione rispetto agli studi con cui confrontare i risultati. $\mathrm{Ci}$ sono, tuttavia, tre costrutti sui quali è opportuno aggiungere qualche osservazione, e cioè quelli con essere come verbo all'infinito, con il clitico si (verbi pronominali,

\footnotetext{
5 Se questa è l'interpretazione esatta, si capisce perché spesso il fenomeno è visto come una caratteristica della più recente evoluzione dell'italiano. Resta comunque il fatto che, però, si tratta della realizzazione sempre più diffusa di un fenomeno di lungo periodo.
} 
costruzioni passive o costruzioni impersonali) o con i verbi al passivo. Anche a questo proposito, confrontando il nostro corpus con quello di Boysen (1977), si nota, globalmente, un'ulteriore estensione dell'uso di avere, in linea con l'evoluzione generale del fenomeno (tranne che nel caso del passivo, tab. 4, per il quale Boysen, su 16 esempi, non ne aveva trovato nessuno con essere, mentre nel nostro corpus, su 64 casi di passivo, ne compaiono 3 con essere: ma, dato il basso numero di esempi, si tratta di una differenza scarsamente rilevante).

\begin{tabular}{|l|l|l|l|l|}
\hline ausiliare & potere & dovere & volere & totale \\
\hline avere & $96,77 \%$ & $92,31 \%$ & $100,00 \%$ & $95,31 \%$ \\
\hline essere & $3,23 \%$ & $7,69 \%$ & $0,00 \%$ & $4,69 \%$ \\
\hline totale & $100,00 \%$ & $100,00 \%$ & $100,00 \%$ & $100,00 \%$ \\
\hline
\end{tabular}

Tab. 4 - Distribuzione in percentuale degli ausiliari in dipendenza dai tre verbi modali nelle costruzioni passive

Nel caso dei verbi pronominali, o nei verbi costruiti con si passivante o impersonale (tab. 5), possiamo ripetere quanto avevamo osservato a proposito dei dati globali: nel confronto con Boysen (1977), risulta un aumento dell'uso di avere (ma con un incremento ben più sensibile, dato che si passa dal $64,29 \%$ dello spoglio di Boysen al nostro $75,43 \%$, con una differenza di oltre 11 punti percentuali); inoltre, nel nostro corpus risulta confermato che potere risulta più resistente alla diffusione di avere rispetto a dovere e volere, mentre anche in questi contesti specifici nel corpus di Boysen potere risulta favorire l'uso di avere più di dovere.

\begin{tabular}{|l|l|l|l|l|}
\hline ausiliare & potere & dovere & volere & totale \\
\hline avere & $66,91 \%$ & $78,77 \%$ & $99,06 \%$ & $75,43 \%$ \\
\hline essere & $33,09 \%$ & $21,23 \%$ & $0,94 \%$ & $24,57 \%$ \\
\hline totale & $100,00 \%$ & $100,00 \%$ & $100,00 \%$ & $100,00 \%$ \\
\hline
\end{tabular}

Tab. 5 - Distribuzione in percentuale degli ausiliari in dipendenza dai tre verbi modali con verbi pronominali (o con si passivante o impersonale)

Ben più stabile appare il comportamento dell'ausiliare quando il verbo all'infinito retto dal modale è essere (tab. 6). Già Boysen (1977) aveva trovato, in questo contesto, un'assoluta preminenza di avere $(89,39 \%$, che aumenta leggermente nel nostro corpus, raggiungendo il 92,93\%) e la sequenza dei verbi modali è già quella che appare preminente nel corpus più ampio, e cioè potere - dovere - volere: come dire che l'evoluzione più recente è iniziata precocemente nei contesti nei quali il verbo all'infinito implicato nel costrutto modale è essere. 


\begin{tabular}{|l|l|l|l|l|}
\hline ausiliare & potere & dovere & volere & totale \\
\hline avere & $89,63 \%$ & $93,14 \%$ & $100,00 \%$ & $92,93 \%$ \\
\hline essere & $10,37 \%$ & $6,86 \%$ & $0,00 \%$ & $7,07 \%$ \\
\hline totale & $89,63 \%$ & $93,14 \%$ & $100,00 \%$ & $92,93 \%$ \\
\hline
\end{tabular}

Tab. 6 - Distribuzione in percentuale degli ausiliari in dipendenza dai tre verbi modali con essere all'infinito

Un parametro fondamentale è, infine, costituito dalla provenienza geografica degli scrittori, secondo le tre macroaree del Nord, della Toscana e del Sud ${ }^{66}$ Skytte (1983: 93-94) ha individuato la pertinenza dell'origine regionale degli scriventi a proposito del fenomeno, parallelo a quello qui trattato, della posizione del clitico con i verbi modali.

Telve (2007) ha segnalato, in tutta la storia dell'italiano, le tendenze differenti che separano gli scrittori toscani dai non toscani, per i quali l'impiego dell'ausiliare essere sembra risultare un uso ereditato per via letteraria, mentre la forma più naturale, perché supportata dal sostrato dialettale, sarebbe l'ausiliare avere.

Lo scarto degli scrittori toscani dai non toscani appare evidente. Non solo nei toscani contemporanei (tab. 7) la percentuale di uso dell'ausiliare avere è meno elevata che negli scrittori di altra provenienza, anche se avere si conferma l'ausiliare più impiegato (sia pure, con potere e dovere, di poco); ma nei toscani pare crearsi una contrapposizione dicotomica tra potere e dovere da una parte (si noti, verbi con altri comportamenti sintattici simili, in quanto entrambi verbi a sollevamento) e volere (verbo a controllo) dall'altra: la percentuale di ricorso ad avere con i primi due modali è, nei toscani, sostanzialmente identica.

\begin{tabular}{|l|l|l|l|l|}
\hline ausiliare & potere & dovere & volere & totale \\
\hline avere & $53,26 \%$ & $53,93 \%$ & $74,29 \%$ & $56,94 \%$ \\
\hline essere & $46,74 \%$ & $46,07 \%$ & $25,71 \%$ & $43,06 \%$ \\
\hline totale & $100,00 \%$ & $100,00 \%$ & $100,00 \%$ & $100,00 \%$ \\
\hline
\end{tabular}

Tab. 7 - Distribuzione in percentuale degli ausiliari in dipendenza dai tre verbi modali negli scrittori toscani

Per quel che riguarda gli scrittori settentrionali e quelli meridionali, se esaminiamo l'uso degli ausiliari globalmente, cioè senza distinzione tra modali, ricaviamo che la distribuzione è analoga, con una percentuale che si aggira, in entrambi i sottocampioni, sul 76\%. I dati prendono una fisionomia almeno parzialmente diversa se guardiamo, però, alla distribuzione modale per modale. Per gli scrittori meridionali (tab. 8) possiamo fare una considerazione analoga a quella fatta per i toscani: il compor- 
tamento di potere e dovere è sostanzialmente identico, anche se si colloca su una soglia ben più alta di uso di avere rispetto alla Toscana.

\begin{tabular}{|l|l|l|l|l|}
\hline ausiliare & Potere & Dovere & volere & totale \\
\hline avere & $72,41 \%$ & $73,13 \%$ & $96,40 \%$ & $76,71 \%$ \\
\hline essere & $27,59 \%$ & $26,87 \%$ & $3,60 \%$ & $23,29 \%$ \\
\hline totale & $100,00 \%$ & $100,00 \%$ & $100,00 \%$ & $100,00 \%$ \\
\hline
\end{tabular}

Tab. 8 - Distribuzione in percentuale degli ausiliari in dipendenza dai tre verbi modali negli scrittori meridionali

La stessa cosa non può dirsi per gli scrittori settentrionali, nei quali l'uso dell'ausiliare con i tre modali presenta valori considerevolmente diversi, collocando l'uso di avere con potere circa 10 punti più in basso di quanto non accada con dovere; mentre volere si distanzia di ulteriori 20 punti (tab. 9).

\begin{tabular}{|l|l|l|l|l|}
\hline ausiliare & Potere & dovere & volere & totale \\
\hline avere & $67,59 \%$ & $78,83 \%$ & $98,64 \%$ & $76,22 \%$ \\
\hline essere & $32,41 \%$ & $21,17 \%$ & $1,36 \%$ & $23,78 \%$ \\
\hline totale & $100,00 \%$ & $100,00 \%$ & $100,00 \%$ & $100,00 \%$ \\
\hline
\end{tabular}

Tab. 9 - Distribuzione in percentuale degli ausiliari in dipendenza dai tre verbi modali negli scrittori settentrionali

Possiamo dunque dire che la considerazione fatta a commento della tab. 3, relativamente alla differenziazione dei tre modali per quel che riguarda l'abbinamento con l'ausiliare nei tempi composti, si deve principalmente al peso che nel corpus hanno gli scrittori settentrionali. La tripartizione che, a prima vista, appare tipica dell'italiano odierno riguarda, dunque, solo l'italiano settentrionale; nelle altre zone d'Italia si nota una bipartizione (potere e dovere vs volere), anche se assestata, nelle due zone, su valori diversi.

\section{UNO SGUARDO SUL PARLATO}

Visti i risultati dell'analisi del corpus di letteratura del dopoguerra, e il confronto con studi analoghi già pubblicati, ci si può attendere che nel parlato l'uso prevalente consista nell'utilizzo dell'ausiliare avere nei tempi composti dei verbi modali, qualsiasi sia il verbo all'infinito presente nel costrutto. Purtroppo, come si è detto, non mi risultano disponibili corpora di italiano parlato di dimensioni corrispondenti a quello letterario utilizzato finora. Pertanto, non è possibile affiancare a quella proposta finora una descrizione altrettanto dettagliata. Utilizzando il corpus 
del LIP, abbiamo isolato solo 32 contesti di uso di verbi modali con verbi inaccusativi.

Sorprendentemente, la distribuzione degli ausiliari (tab. 10) presenta una configurazione del tutto opposta rispetto a quella emersa dallo spoglio dei corpora letterari: prevale l'uso di essere sia complessivamente, sia per potere sia per dovere (dovere presenta il tasso più basso di ricorso ad avere). Solo volere mostra la prevalenza di avere.

\begin{tabular}{|l|l|l|l|l|}
\hline ausiliare & Potere & Dovere & volere & totale \\
\hline avere & $28,57 \%$ & $25,00 \%$ & $66,67 \%$ & $34,38 \%$ \\
\hline essere & $71,43 \%$ & $75,00 \%$ & $33,33 \%$ & $65,63 \%$ \\
\hline totale & $100,00 \%$ & $100,00 \%$ & $100,00 \%$ & $100,00 \%$ \\
\hline
\end{tabular}

Tab. 10 - Distribuzione in percentuale degli ausiliari in dipendenza dai tre verbi modali nel corpus del LIP (De Mauro et alii 1993)

Questo risultato sorprendente ha bisogno di una conferma o di una smentita dall'analisi di un corpus più consistente, di cui attualmente non sono in condizioni di disporre.

\section{CONCLUSIONI}

Lo spoglio di un ampio corpus di letteratura contemporanea della seconda metà del Novecento ci ha permesso di confermare, e consolidare, con la forza di un'ampia messe di dati, le conclusioni già disponibili sull'uso dell'ausiliare nei tempi composti dei verbi modali legati a verbi inaccusativi.

Contrariamente a quanto implicitamente assunto dalle grammatiche normative, e anche da alcune sintesi sull'italiano contemporaneo, l'ausiliare di gran lunga più usato nei costrutti presi in esame è avere. La prevalenza di avere non è una caratteristica dell'italiano contemporaneo, ma certamente il suo uso si sta sempre più ampliando. A questa conclusione porta il confronto tra lo spoglio del corpus di Boysen (1977) e il nostro, fondato su basi simili, ma ovviamente più esteso cronologicamente.

Nel breve periodo risulta mutata la familiarità con l'ausiliare avere dei diversi modali: mentre volere ha sempre mostrato una maggiore predisposizione all'ausiliare avere, l'inversione dell'ordine tra dovere (oggi più legato ad avere) e potere è un fatto recente.

La prevalenza di avere rispetto ad essere mostra valori quantitativi e configurazioni diverse nelle diverse zone d'Italia. Per il dato quantitativo, c'è una contrapposizione tra Toscana (dove la propensione all'uso di avere è più ridotta) e il resto d'Italia; circa la configurazione, Toscana e Italia meridionale mostrano un comportamento analogo di potere e dovere, che presentano un ricorso ad avere quantitativamente molto simile, in contrapposizione a volere, molto più orientato all'uso 
di tale ausiliare; 1'Italia settentrionale mostra, invece, di aver costituito una scala di propensione all'uso di avere che vede potere nella posizione più bassa, dovere in quella mediana, volere, ancora una volta, in quella più alta.

Questa situazione sostanzialmente non dipende dal verbo implicato nel complesso verbale. In particolare, l'uso dell'ausiliare quando il verbo all'infinito è essere appare anticipare le tendenze più generali; già nello spoglio di Boysen (1977) il ricorso ad avere era assolutamente preminente e si verificava la scala di propensione potere dovere - volere, che per gli altri verbi emerge, soprattutto ad opera degli scrittori settentrionali, solo nel corpus che tiene conto anche degli anni più recenti.

Contraria alle attese è la distribuzione degli ausiliari che emerge dall'esame di un corpus di italiano parlato: risulta prevalere l'uso di essere. La ristrettezza del corpus ci induce, però, a sospendere il giudizio, in attesa dello spoglio di corpora più ampi.

In conclusione, possiamo sostenere con certezza che la prescrizione delle grammatiche che stabiliscono la preferenza, dal punto di vista normativo, dell'uso dell'ausiliare essere con i verbi modali che si accompagnano a verbi inaccusativi è assolutamente anacronistica e va in direzione contraria rispetto a una chiara linea evolutiva della storia dell'italiano. 


\section{Bibliografia}

Boysen, Gerhard (1977) «L'emploi des verbes auxiliaries 'essere' et 'avere' avec les verbes modaux en italien.» Studia Neophilologica XLIX, 287-309.

Coletti, Vittorio (1993) Storia dell'italiano letterario. Torino: Einaudi.

Cortelazzo, Michele A. (2007a) «Evoluzione della lingua, percezione del cambiamento, staticità della norma.» In: E. Pistolesi (a cura di), Lingua scuola e società. I nuovi bisogni comunicativi nelle classi multiculturali. Atti del Convegno organizzato dall'Istituto Gramsci del Friuli Venezia Giulia con il patrocinio dell'Università degli Studi di Trieste, Trieste, 6 - 7 ottobre 2006. Trieste: Istituto Gramsci del Friuli Venezia Giulia, 47-55.

Cortelazzo, Michele A. (2007b) «La perifrasi progressiva in italiano è un anglicismo sintattico?» In: Allievi padovani (a cura di), Studi in onore di Pier Vincenzo Mengaldo per i suoi settant'anni. Firenze: Sismel, Edizioni del Galluzzo, 1753-1764.

D'ACHILle, Paolo. (2003) L'italiano contemporaneo. Bologna: il Mulino.

Della Valle, Valeria/Pietro Trifone (a cura di) (2007) Studi linguistici per Luca Serianni. Roma: Salerno.

De Mauro, Tullio/Federico Mancini/Massimo Vedovelli/Miriam Voghera (a cura di) (1993) Lessico di frequenza dell'italiano parlato. Milano: ETAS.

FochI, Franco (1967) L'italiano facile. Guida allo scrivere e al parlare. Milano: Feltrinelli.

RenzI, Lorenzo/Giampaolo SALVI/Anna CARDINALETTI (2001) Grande grammatica italiana di consultazione. Vol. II [Nuova edizione]. Bologna: il Mulino.

SATtA, Luciano (1989) Matita rossa e blu. Lo stato della lingua italiana nell'esame spietato ma scherzoso compiuto su 110 scrittori contemporanei. Milano: Bompiani.

Serianni, Luca (2006) Prima lezione di grammatica. Roma/Bari: Laterza.

Severgnini, Beppe (2007) L'italiano. Lezioni semiserie. Milano: Rizzoli.

SKYTTE, Gunver (1983) La sintassi dell' infinito in italiano moderno. Kobenhavn: Munksgaard.

TAvoni, Mirko (2002) «Caratteristiche dell'italiano contemporaneo e insegnamento della scrittura.» In: F. Bruni/T. Raso (a cura di), Manuale dell'italiano professionale. Teoria e didattica. Bologna: Zanichelli, 139-152.

Telve, Stefano (2007) «Essere o avere? Sull'alternanza degli ausiliari coni modali potuto, voluto (e dovuto) davanti a infiniti inaccusativi in italiano antico e moderno.» In V. Della Valle/P. Trifone (a cura di), Studi linguistici per Luca Serianni. Roma: Salerno, 313-325. 


\section{Riassunto \\ EVOLUZIONE DELLA LINGUA E STATICITÀ DELLA NORMA NELL'ITALIANO \\ CONTEMPORANEO: GLI AUSILIARI NEI COSTRUTTI CON VERBI MODALI}

Tra i fenomeni che vengono attribuiti alla recente evoluzione dell'italiano (e che vengono stigmatizzati dalle grammatiche normative) vi è l'uso generalizzato dell'ausiliare avere nei tempi composti dei verbi modali, anche quando il verbo modale regge un verbo all'infinito che nei tempi composti richiede l'ausiliare essere.

Studi recenti hanno già mostrato che, nell'uso, l'impiego di avere è prevalente rispetto all'uso di essere e che si tratta di un fenomeno che percorre tutta la storia dell'italiano.

Queste conclusioni sono state confermate e rafforzate dall'analisi di un corpus formato da 116 romanzi italiani pubblicati a partire dal secondo dopoguerra.

\section{Povzetek}

\section{RAZVOJ JEZIKA IN STATIČNOST NORME V SODOBNI ITALIJANŠČINI: POMOŽNIKI V KONSTRUKCIJAH Z MODALNIMI GLAGOLI}

Med pojavi, ki naj bi bili značilni za novejši razvoj italijanščine (in ki jih normativne slovnice stigmatizirajo), je posplošena raba pomožnika avere $\mathrm{v}$ sestavljenih časih modalnih glagolov tudi takrat, kadar se modalni glagol veže z glagolom v nedoločniku, ki v sestavljenih časih zahteva pomožnik essere.

Nedavne študije so že pokazale, da v rabi prevladuje pomožnik avere $\mathrm{v}$ primerjavi s pomožnikom essere in da gre za pojav, ki je prisoten skozi vso zgodovino italijanščine.

Te zaključke je potrdila in utrdila analiza korpusa, ki ga sestavlja 116 italijanskih romanov, objavljenih po drugi svetovni vojni. 